\title{
Control prehospitalario de la hemorragia en pacientes de trauma: una estrategia de prevención secundaria factible para países de bajos y medianos ingresos
}

\author{
Prehospital control of hemorrhage in trauma patients: a feasible secondary \\ prevention strategy in countries of low and median income \\ Claudia Patricia Orlas¹, Ramiro Manzano-Núñez², Juan Pablo Herrera², \\ Alberto Federico García ${ }^{3}$, Diana C. Muñoz ${ }^{4}$, Julián Chica ${ }^{1}$, Camilo José Salazar ${ }^{5}$, \\ María Alejandra Londoño ${ }^{5}$, Juan Esteban Ruiz ${ }^{5}$, Juan José Meléndez ${ }^{6}$, José Julián Serna 6 , \\ Edison Angamarca ${ }^{6}$, Michael W. Parra ${ }^{7}$, Carlos Alberto Ordóñez ${ }^{8}$
}

1 Médico rural en investigación, Centro de Investigaciones Clínicas, Fundación Valle del Lili, Cali, Colombia

2 Research fellow, Center for Surgery and Public Health, Department of Surgery, Brigham \& Women's Hospital, Harvard Medical School and Harvard T. H. Chan School of Public Health, Boston, MA, USA

3 Cirujano de Trauma y Emergencias, Fundación Valle del Lili; docente de Cirugía General, Universidad del Valle, Cali, Colombia

4 Cirujana general, Sección de Cirugía de Trauma y Emergencias, Fundación Valle del Lili, Cali, Colombia

5 Estudiante de Medicina, Universidad ICESI, Cali, Colombia

6 Fellow de Cirugía de Trauma y Emergencias, Universidad del Valle, Cali, Colombia

7 MD, FACS; Department of Trauma Critical Care, Broward General Level I Trauma Center, Fort Lauderdale, FLA, USA

8 MD, FACS; jefe, Sección de Cirugía de Trauma y Emergencias, Fundación Valle del Lili; docente de Cirugía General, Universidad del Valle, Cali, Colombia

Segundo puesto en el Simposio Nacional del Residente Quirúrgico. XLIV Congreso Nacional "Avances en Cirugía". Cartagena, Colombia, agosto de 2018

\section{Resumen}

Introducción. La hemorragia no controlada es la principal causa prevenible de muerte en trauma. No hay precedentes locales que estimen el efecto de estrategias de prevención que involucren al público en la atención inicial de las víctimas.

Objetivo. Evaluar si es factible implementar programas de entrenamiento para el control prehospitalario básico del sangrado en pacientes de trauma, en un país de bajos a medianos ingresos.

Métodos. Cirujanos y estudiantes de medicina se encargaron de desplegar el curso "Stop the bleed" en estudiantes universitarios del suroccidente colombiano. Se hizo una evaluación antes y después de hacer el entrenamiento. Se usó la prueba t de Student y un análisis de regresión logística ordinal, para determinar los factores que estuvieron asociados a obtener mejores puntajes en la evaluación del curso.

Resultados. Se entrenaron 265 estudiantes, con una edad media de 2I,4 \pm 4 años, de los cuales 136 (5I,5\%) eran mujeres. Después de recibir el entrenamiento un participante tuvo I5,6 veces la oportunidad de obtener mayor puntaje de calificación con respecto al periodo preentrenamiento [IC ${ }_{95 \%}$ : I5,I-I6,2 (p<0,00I)], 99,4 y 95,2 \%, respectivamente, estarían dispuestos a tomar medidas y colocar un torniquete a una víctima de sangrado ( $<<0,00 I)$.

Fecha de publicación: 8/06/2018 - Fecha aceptación: 28/08/2018

Correspondencia: Claudia Patricia Orlas, MD

Carrera 98 N 18-49, Centro de Investigaciones Clínicas, Fundación Valle del Lili, torre 7, piso 3, Cali, Colombia - Teléfono: (317) 894-8698 Correo electrónico: claudiapatriciaorlas@gmail.com

Citar: Orlas CP, Manzano-Núñez R, Herrera JP, García AF, Chica J, Salazar CJ, et al. Control prehospitalario de la hemorragia en pacientes de trauma: una estrategia de prevención secundaria factible para países de bajos y medianos ingresos. Rev Colomb Cir. 2018;33:371-9. https://doi.org/10.30944/20117582.84

Este es un artículo de acceso abierto bajo una Licencia Creative Commons - BY-NC-ND https://creativecommons.org/licenses/by-nc-nd/4.0/deed.es 
Conclusión. Es factible para los países con recursos limitados hacer el entrenamiento en control básico del sangrado. Este se puede llevar a cabo de manera eficaz, tanto por instructores con un bagaje amplio en técnicas de control del sangrado como por instructores con menor nivel de formación, pero con un entrenamiento adecuado. Palabras clave: hemorragia; heridas y traumatismos; urgencias médicas; prevención y control; educación de la población; salud pública.

\begin{abstract}
Background: uncontrolled hemorrhage is the leading cause of preventable death in trauma. There are no local precedents that estimate the effect of prevention strategies that involve the public in the initial care of victims. Our objective was to evaluate if it is feasible to implement training programs for the basic control of prehospital bleeding in trauma patients, in a low to a middle-income country.

Methods: Surgeons and medical students deploying the Stop the bleed course in a Colombian southwestern university, evaluation was performed before and after the training. A T-test and an ordinal logistic regression analysis were used to determine the factors associated with better scores in the course evaluation.

Results: of the 265 students, were women 136 (51.5\%), age was 21.4 \pm 4 . After receiving the training, a participant had I5.6 times the chance of obtaining a higher score than the pre-training period [95\% CI: I5.I-I6.2 (p <0.00I)]. 99.4\% and $95.2 \%$ respectively, would be willing to take actions and place a tourniquet on a bleeding victim $(\mathrm{p}<0.00 \mathrm{I})$. Conclusion: in a low to a middle-income country is feasible to perform the Bleeding Control Basic training. This can be done effectively by instructors with a broad background in bleeding control techniques as well as by instructors with less experience, but with adequate training.
\end{abstract}

Key words: hemorrhage; wounds and injuries; emergencies; prevention \& control; population education; public health.

\section{Introducción}

La hemorragia no controlada es la principal causa de muerte prevenible en trauma, y la intervención temprana para controlar el sangrado puede incrementar la probabilidad de supervivencia ${ }^{\mathrm{I}-3}$. En la actualidad, es una prioridad para el American College of Surgeons preparar al público en conocimientos para el control del sangrado frente a desastres de carácter local y nacional ${ }^{4}$.

El programa Stop the bleed lanzado por la Casa Blanca en el 2015, pretende informar, educar y empoderar a la población civil para que actúen como respondedores inmediatos para controlar la hemorragia que pone en riesgo la vida, en el sitio en donde se produce el trauma ${ }^{5,6}$. En respuesta a esta iniciativa, el Trauma Committee del American College of Surgeons, en conjunto con la National Association of Emergency Medical Technicians en Estados Unidos, desarrollaron el Bleeding control course: $B$-Con (curso de control básico del sangrado), el cual está dirigido a público lego ${ }^{5}$. El curso ha tenido una gran acogida, con más de I6.00o instructores registrados y un estimado de I00.000 a 150.000 ciudadanos entrenados por año ${ }^{6}$.
En Colombia, la mortalidad por trauma en una unidad de cuidados intensivos de un centro de trauma de alto volumen, alcanza hasta el Io $\%{ }^{7}$. El país está entre los 20 países con mayor tasa de homicidios a nivel mundial ${ }^{8}$. A pesar de contar con instituciones con vasta experiencia en el manejo del trauma, no existe un consenso de carácter local o nacional que especifique cuál es el rol de la población como respondedores iniciales en los sitios de trauma o si es factible implementar estrategias educativas frente a esta problemática.

Nuestra hipótesis es que es factible implementar programas de entrenamiento en el control básico del sangrado en trauma en los países de bajos a medianos ingresos, y la calidad del mismo no está influenciada por el bagaje de quienes dirijan el entrenamiento.

En este sentido, los objetivos de este estudio fueron: I) evaluar si es factible implementar programas de entrenamiento para el control prehospitalario básico del sangrado en pacientes de trauma en un país de bajos a medianos ingresos, y 2) estimar si existe asociación entre el tipo de 
entrenador, y los puntajes teóricos y prácticos obtenidos de la evaluación del curso.

\section{Materiales y métodos Diseño del estudio}

Este es un estudio cuasiexperimental, aprobado por el comité de revisión institucional de un centro de trauma de nivel I de Cali en febrero del 20I8. El objetivo primario fue evaluar si es factible implementar programas de entrenamiento para el control prehospitalario del sangrado en pacientes de trauma en un país de bajos a medianos ingresos. El objetivo secundario fue estimar si existe asociación entre el tipo de entrenador, y los puntajes teóricos y prácticos obtenidos de la evaluación del curso.

\section{Participantes}

Los participantes fueron los estudiantes de pregrado de dos universidades adscritas en Cali, una universidad pública y una universidad privada.

La convocatoria para el entrenamiento estuvo abierta tanto para estudiantes pertenecientes a programas de salud como para los de otras áreas (por ejemplo, ingenierías, humanidades, ciencias sociales y económicas). La participación para diligenciar los instrumentos con las variables de interés para el estudio, se hizo de forma voluntaria y no requirió de un consentimiento informado.

El grupo de instructores considerados para impartir el curso, estuvo conformado por cirujanos generales y estudiantes de medicina pertenecientes a la Liga de Trauma y Emergencias del Pacífico (LITEP), un semillero de investigación adscrito a la Sociedad Panamericana de Trauma, integrado por estudiantes en diferentes niveles de formación de medicina, cirugía general, cirugía de trauma y emergencias. Todos los instructores recibieron entrenamiento por parte del American College of Surgeons y se encontraban registrados en el curso B-Con.

\section{Curso "Bleeding control" (B-Con) - Control básico del sangrado}

En respuesta a la masacre ocurrida en la escuela primaria de Sandy Hook en Connecticut, EE. UU., en la que, por lo menos, murieron 28 personas, el American College of Surgeons hizo parte del grupo de expertos en trauma conformado por representantes de la comunidad, gobierno federal y organizaciones de respuesta en emergencias, entre otros, para conformar el consenso Hartford. Este grupo describe al público como respondedor inmediato y la clave para proveer un punto de corte en el control de la hemorragia, con el objetivo de disminuir el número de muertes prevenibles por trauma.

En el 20I5, la Casa Blanca hizo el lanzamiento de Stop the bleed, una campaña con varios programas educativos que fueron emergiendo para enseñarle al público los principios básicos para controlar el sangrado. El uso del torniquete en el campo de guerra en Afganistán e Irak, a menudo por miembros del servicio sin formación en medicina, ha salvado entre I.00o y 2.000 vidas, lo cual sentó un precedente para que, en el diseño de las estrategias para impartir el curso, se considerara su uso en la población civil ${ }^{9-\mathrm{II}}$.

El entrenamiento tiene un componente teórico seguido de un componente práctico. Este programa se enfoca en la respuesta inmediata al sangrado, el reconocimiento del sangrado que pone en riesgo la vida y las formas apropiadas para detener el sangrado. Los tres pilares más importantes están contenidos en el "ABC del sangrado": A: alerta, llamar para activar el sistema de emergencias; B: Bleeding (sangrado) -encontrar la herida que lo provoca, y C: compresión, aplicar presión para detener el sangrado, por medio de una de las tres técnicas: cubrir la herida con un trapo limpio aplicando presión directa con las dos manos, usando un torniquete, o empaquetando la herida con gasas o un trapo limpio y luego aplicar presión con ambas manos ${ }^{12}$.

El entrenamiento completo (teórico-práctico) tiene una duración de una hora. La práctica se llevó a cabo con un equipo de simulación de extremidades amputadas o heridas profundas, implementado con torniquetes y gasas.

\section{Protocolo del estudio}

Los participantes fueron convocados en una fecha para recibir el curso en horarios diferentes a lo largo de un día. Hubo auditorios disponibles 
clasificados de acuerdo con el tipo de entrenador a cargo de impartir la clase, los cirujanos generales o los estudiantes de medicina. Los participantes se asignaron por subgrupos a cada instructor.

El entrenamiento contempló cinco fases:

I. Diligenciamiento de un cuestionario con las variables sociodemográficas de interés para el estudio, y evaluación subjetiva de la capacidad de respuesta de los participantes frente a una víctima de trauma que sufre sangrado.

2. Completar un examen teórico antes del entrenamiento para evaluar los conocimientos sobre las técnicas básicas para controlar el sangrado.

3. Clase teórica.

4. Clase práctica en modelos de simulación.

5. Evaluación teórica y práctica posterior al entrenamiento.

Como instrumento de evaluación antes y después del entrenamiento, se usó la escala de Liker que consta de cinco puntos, para indicar la probabilidad de desempeñar las siguientes tareas:

I. tomar medidas si fuera la única persona disponible para ayudar a una víctima de sangrado,

2. aplicar presión correctamente para controlar el sangrado, y

3. colocar un torniquete a una víctima con sangrado.

La respuesta dada en los términos "Muy probable" y "Probable", se combinó para indicar la probabilidad de completar las tareas especificadas en las preguntas.

Con la evaluación teórica se determinó el conocimiento de los participantes de los componentes del "ABC del sangrado". Para la evaluación práctica, se presentó una situación a cada uno de los participantes, en la cual debían responder en el contexto de una situación de urgencia frente a una víctima de sangrado por fuera de un hospital. El entrenamiento y la evaluación se hicieron de manera independiente y ningún cirujano o estudiante podía intervenir sobre el curso de la clase o la evaluación de los grupos asignados.

\section{Análisis estadístico}

Para el análisis estadístico se utilizó el programa Stata I4. $^{\mathrm{TM}}$. La evaluación de la distribución de la normalidad de las variables, se hizo con el test de Shapiro Wilk, asumiendo normalidad para aquellas variables que presentaran un valor de $\mathrm{p}$ mayor de 0,05 . Las variables continuas se presentaron en medias y desviación estándar; y las variables categóricas, en números enteros y porcentajes.

Para el análisis global del puntaje obtenido en la escala de Likert de la probabilidad general de completar las tareas para controlar el sangrado, se presentó la proporción de los sujetos que estarían dispuestos a completar las tareas asignadas antes y después de recibir el entrenamiento; estos resultados se compararon después mediante la prueba t de Student para muestras pareadas, y se estableció un nivel de significancia estadística menor de 0,05 .

El estudio de los factores que se asociaron a tener una mayor probabilidad de éxito en los puntajes obtenidos dentro de la evaluación de la eficacia del entrenamiento, se ajustaron de acuerdo con el periodo (antes o después del entrenamiento), el tipo de entrenador (cirujano o estudiante de medicina) y la probabilidad de recibir el entrenamiento estándar (cirujano) versus estudiantes, con un modelo de regresión logística ordinal utilizando el software estadístico RGui, con el que se estableció un nivel de significación menor de 0,05 para las variables que guardaron asociación con los factores para los que se ajustó el modelo.

\section{Resultados}

En total, 265 participantes completaron el entrenamiento, cuya edad media fue 2I,4 años, $y$ I36 (5I,5\%) eran mujeres. La mayoría, I52 (57,3\%), procedía de la ciudad donde se hizo el entrenamiento, 32 (I2,I \%) pertenecían a estratos socioeconómicos I y 2 , aunque la mayoría (I26; 47,9\%) se ubicó entre en los estratos 3 y 4 . De los estudiantes, 42 ( $15,8 \%)$ pertenecían a una universidad pública y $223(84, \mathrm{I} \%)$ procedían de universidades privadas. Más de dos tercios $(\mathrm{n}=250 ; 94,3 \%)$ de los 
participantes hacían parte de un programa de la Facultad de Ciencias de la Salud y $80(30,1 \%)$ manifestaron que habían asistido previamente a otros cursos relacionados con el control prehospitalario del sangrado (tabla 1).

Para evaluar la efectividad de los medios de difusión usados para promocionar el curso, se preguntó a los participantes si tenían algún conocimiento sobre la campaña, y $90(36,9 \%)$ manifestaron haber recibido información previa al respecto. Las dos herramientas utilizadas con más frecuencia fueron la promoción hecha por la Liga de Trauma y Emergencias del Pacífico (LITEP) y las redes sociales. En cuanto al tipo de entrenador, I2I (45,6\%) estuvieron a cargo de un cirujano general y, I 44 (54,3\%), de estudiantes de medicina (tabla 2).

Para evaluar las actitudes de los participantes antes y después de recibir el entrenamiento, se estratificó la población en dos grupos, quienes

Tabla 1. Características de los participantes en el estudio $(n=265)$

\begin{tabular}{lc}
\hline \multicolumn{1}{c}{ Variables sociodemográficas } & $\mathbf{n}(\%)$ \\
\hline Edad, media (DE) & $21,4(4,0)$ \\
Sexo femenino & $136(51,5)$ \\
Procedencia & \\
Santiago de Cali & $152(57,3)$ \\
Municipio diferente a Cali & $37(13,9)$ \\
Otro departamento de Colombia & $74(27,9)$ \\
País diferente a Colombia & $2(0,7)$ \\
Estrato socioeconómico & \\
Estratos 1 y 2 & $32(12,1)$ \\
Estratos 3 y 4 & $126(47,9)$ \\
Estratos 5 y 6 & $105(39,8)$ \\
Tipo de universidad & \\
Universidad pública & $42(15,8)$ \\
Universidad privada & $223(84,1)$ \\
Tipo de facultad & \\
Facultad de Ciencias de la Salud & $250(94,3)$ \\
Otras facultades & $15(5,7)$ \\
Entrenamiento médico previo & \\
Cursos control del sangrado (\%)* & $80(30,1)$ \\
\hline DE: desviación estándar & \\
*Haber realizado otros cursos en técnicas para control del sangrado
\end{tabular}

Tabla 2. Conocimiento de la campaña Stop the bleed y entrenador asignado $(n=265)$

\begin{tabular}{lc}
\hline $\begin{array}{l}\text { Conocimiento previo sobre la campaña } \\
\text { Stop the bleed (STB) }\end{array}$ & $\mathbf{n}(\%)$ \\
\hline Conocimiento de la campaña STB & $98(36,9)$ \\
Medio por el que conoció la campaña & \\
Promoción LITEP* & $86(32,7)$ \\
Cartelera o afiche & $22(8,3)$ \\
Redes sociales & $82(31,1)$ \\
Información de otro compañero & $73(27,7)$ \\
Tipo de entrenador & \\
Cirujano general & $121(45,6)$ \\
Estudiante de medicina & $144(54,3)$ \\
\hline
\end{tabular}

* LITEP: Liga de Trauma y Emergencias del Pacífico

habían recibido algún tipo de entrenamiento previamente y aquellos que no tenían ningún entrenamiento (tabla 3). En el primer grupo, 98,7 \% manifestó que tomaría alguna medida para ayudar a una víctima que está sangrado, antes y después del entrenamiento, sin diferencias estadísticamente significativas entre los dos periodos de evaluación $(\mathrm{p}=0,089)$. Antes del entrenamiento, $65(82,2 \%)$ refirieron que aplicarían presión correctamente para controlar el sangrado, número que aumentó a 75 (98,7 \%) después del entrenamiento, una diferencia estadísticamente significativa $(\mathrm{p}<\mathrm{O}, \mathrm{OoI})$ La proporción de participantes que estarían dispuestos a colocar un torniquete a una víctima con sangrado, aumentó de manera significativa después de haber sido entrenados: antes, 34 (43,0 \%), y después, 70 (92,I \%) (p<0,ooI). Para el segundo grupo, la proporción de participantes que estarían dispuestos a tomar medidas, aplicar presión sobre una herida para controlar el sangrado y colocar un torniquete, aumentó de manera significativa después del entrenamiento (tabla 3).

Para analizar los factores asociados con una mayor probabilidad de éxito después del entrenamiento, se usó un modelo de regresión logística ordinal (tabla 4). Se establecieron tres categorías ajustadas por periodo, tipo de entrenador, y relación entre el cirujano y el estudiante de medicina. 
Tabla 3. Respuestas al cuestionario antes y después del entrenamiento

\begin{tabular}{|c|c|c|c|}
\hline Con entrenamiento previo & $\begin{array}{c}\text { Antes del } \\
\text { entrenamiento* } \\
n(\%)\end{array}$ & $\begin{array}{l}\text { Después del } \\
\text { entrenamiento* } \\
n(\%)\end{array}$ & p \\
\hline $\begin{array}{l}\text { ¿Tomaría medidas si fuera la única persona } \\
\text { disponible para ayudar una víctima que está } \\
\text { sangrado? }\end{array}$ & $78(98,7)$ & $75(98,7)$ & 0,089 \\
\hline $\begin{array}{l}\text { ¿Cree que aplicaría correctamente la presión } \\
\text { para controlar el sangrado? }\end{array}$ & $65(82,2)$ & $75(98,7)$ & $<0,001$ \\
\hline $\begin{array}{l}\text { ¿Aplicaría un torniquete a una víctima que se } \\
\text { encuentra sangrando? }\end{array}$ & $34(43,0)$ & $70(92,1)$ & $<0,001$ \\
\hline \multicolumn{4}{|l|}{ Sin entrenamiento previo } \\
\hline $\begin{array}{l}\text { ¿Tomaría medidas si fuera la única persona } \\
\text { disponible para ayudar una víctima que está } \\
\text { sangrado? }\end{array}$ & $171(92,4)$ & $166(99,4)$ & $<0,001$ \\
\hline $\begin{array}{l}\text { ¿Cree que aplicaría correctamente la presión } \\
\text { para controlar el sangrado? }\end{array}$ & $83(44,8)$ & $162(97,0)$ & $<0,001$ \\
\hline $\begin{array}{l}\text { ¿Aplicaría un torniquete a una víctima que se } \\
\text { encuentra sangrando? }\end{array}$ & $105(56,7)$ & $159(95,2)$ & $<0,001$ \\
\hline
\end{tabular}

Tabla 4. Análisis de regresión logística ordinal, ajustado por periodo de entrenamiento y tipo de entrenador

\begin{tabular}{|c|c|c|c|}
\hline & \multicolumn{3}{|c|}{ Puntaje de evaluación teórica } \\
\hline & OR & $\mathbf{I C}_{95 \%}$ & pe \\
\hline Tiempo (antes y después del entrenamiento) & 15,6 & $15,1-16,2$ & $<0,001$ \\
\hline $\begin{array}{l}\text { Tipo de entrenador } \\
\text { (Cirujano o estudiante de medicina) }\end{array}$ & 0,82 & $0,80-0,85$ & 0,42 \\
\hline \multirow[t]{3}{*}{ Relación de cirujano y estudiante de medicina } & 0,96 & 0,93-1,02 & 0,93 \\
\hline & \multicolumn{3}{|c|}{$\begin{array}{l}\text { ¿Tomaría medidas si fuera la única persona disponible para ayudar una } \\
\text { víctima que está sangrando? }\end{array}$} \\
\hline & OR & $I \mathbf{C}_{95 \%}$ & $\mathbf{p}^{*}$ \\
\hline Tiempo (antes y después del entrenamiento) & 0,23 & $0,22-0,24$ & $<0,001$ \\
\hline $\begin{array}{l}\text { Tipo de entrenador } \\
\text { (Cirujano o estudiante de medicina) }\end{array}$ & 1,09 & $1,06-1,13$ & 0,75 \\
\hline \multirow[t]{3}{*}{ Relación de cirujano y estudiante de medicina } & 0,71 & $0,67-0,77$ & 0,55 \\
\hline & \multicolumn{3}{|c|}{ ¿Cree que aplicaría correctamente la presión para controlar el sangrado? } \\
\hline & OR & $\mathrm{IC}_{95 \%}$ & $\mathbf{p}^{*}$ \\
\hline Tiempo (antes y después del entrenamiento) & 0,06 & $0,06-0,07$ & $<0,001$ \\
\hline $\begin{array}{l}\text { Tipo de entrenador } \\
\text { (Cirujano o estudiante de medicina) }\end{array}$ & 1,28 & $1,25-1,32$ & 0,30 \\
\hline \multirow[t]{3}{*}{ Relación de cirujano y estudiante de medicina } & 0,56 & $0,54-0,59$ & 0,14 \\
\hline & \multicolumn{3}{|c|}{ ¿Aplicaría un torniquete a una víctima que se encuentra sangrando? } \\
\hline & OR & $\mathbf{I C}_{95 \%}$ & $\mathbf{p}^{*}$ \\
\hline Tiempo (antes y después del entrenamiento) & 0,07 & $0,07-0,08$ & $<0,001$ \\
\hline $\begin{array}{l}\text { Tipo de entrenador } \\
\text { (Cirujano o estudiante de medicina) }\end{array}$ & 0,75 & $0,73-0,77$ & 0,21 \\
\hline Relación de cirujano y estudiante de medicina & 0,55 & $0,53-0,59$ & 0,16 \\
\hline
\end{tabular}

OR: odds ratio 
Respecto al puntaje de la evaluación teórica, después de recibir el entrenamiento un participante tenía 15,6 veces la oportunidad de obtener mayor puntaje de calificación, diferencia estadísticamente significativa con respecto al periodo previo al entrenamiento [IC ${ }_{95 \%}: \mathrm{I} 5, \mathrm{I}-\mathrm{I} 6,2$ $(\mathrm{p}<\mathrm{O}, \mathrm{OoI})]$. Esta diferencia no se observó cuando se hizo el análisis según el tipo de entrenador; es decir, recibir entrenamiento por parte de un cirujano o un de un estudiante de medicina, no aumentó la probabilidad de obtener puntajes más altos en la calificación. En cuanto a la oportunidad de obtener mayor puntaje, según la probabilidad de ser asignado a cualquiera de los dos grupos de entrenamiento, no hubo diferencias estadísticamente significativas entre los dos grupos (odds ratio, $\left.\left.\mathrm{OR}=0,96 ; \mathrm{IC}_{95 \%} \mathrm{O}, 93-\mathrm{I}, \mathrm{O2}\right)(\mathrm{p}=\mathrm{O}, 93)\right]$.

Hubo diferencia estadísticamente significativa entre la afirmación de que aplicarían presión correctamente $\left(\mathrm{OR}=0,06 ; \mathrm{IC}_{95} \% \mathrm{O}, \mathrm{O} 6-\mathrm{O}, \mathrm{O} 7\right)(\mathrm{p}<\mathrm{O}, \mathrm{OOI})$ y la de que colocarían un torniquete $(\mathrm{OR}=0,07$; $\mathrm{IC}_{95 \%}$ 0,07-0,08) ( $\left.\mathrm{p}<\mathrm{O}, \mathrm{OOI}\right)$ para controlar el sangrando, antes y después del entrenamiento. Es decir, recibir el entrenamiento aumentó la probabilidad de que los participantes estuvieran dispuestos a tomar medidas para controlar de manera eficiente la hemorragia.

\section{Discusión}

Los resultados apoyan la hipótesis de que, en países de bajos a medianos ingresos, es factible el entrenamiento en el control básico del sangrado y que su calidad no se ve influenciada por el bagaje de los entrenadores encargados de enseñar las técnicas. El análisis demostró que entre 95,2 \% y $98,7 \%$ de todos los participantes, estarían dispuestos a tomar medidas frente a una víctima de sangrado y que la oportunidad de ser entrenado por alguien que tenga mayor experiencia en el área, como lo es un cirujano con respecto a un estudiante de medicina, no aumentó la probabilidad de obtener mayor puntaje en la evaluación del curso $\left(\mathrm{OR}=0,96 ; \mathrm{IC}_{95 \%} \mathrm{0}, 93-\mathrm{I}, \mathrm{O} 2\right)(\mathrm{p}=0,93)$.

Los hallazgos confirman que el entrenamiento en el control básico del sangrado es efectivo y factible en países de bajos a medianos ingresos, en donde no existen precedentes de esta intervención, a pesar de que históricamente han sido las zonas que tienen mayor prevalencia de trauma. Existe evidencia contundente que apoya el uso de esta intervención como una herramienta para mejorar la capacidad de respuesta del público frente a situaciones que implican la exposición a víctimas de sangrado que pone en riesgo la vida y que requiere la implementación de estrategias oportunas que aumenten la supervivencia ${ }^{3,6}$.

El público que podría representar un punto de corte es aquel que tiene mayor probabilidad de exponerse a incidentes que requieren el auxilio rápido de personas heridas, aquellos que trabajan en áreas de conglomeración masiva como, por ejemplo, colegios, centros comerciales, estadios, aeropuertos y medios de transporte en general, en donde ya son válidas otras estrategias como el uso del desfibribilador externo automático ${ }^{13-15}$.

A pesar de lo expuesto, aunque el curso Stop the bleed enfatiza la necesidad de entrenar personas legas, no describe detalladamente cómo esta estrategia de educación se lleva a cabo efectivamente o los elementos que debe contener para mejorar el proceso de aprendizaje ${ }^{\text {II. }}$.

Es vital evaluar si las estrategias en el entrenamiento son óptimas para garantizar el proceso de aprendizaje; especialmente, en países donde no se pueden reproducir de manera exacta las condiciones en que se difunden cursos como este y es menor la probabilidad de contar con entrenadores que tengan suficientes conocimientos en el área. Por lo tanto, cobra importancia el hallazgo de que personal con menos experiencia esté en condiciones de impartir un curso de igual calidad.

En este estudio se eligió un modelo cuasiexperimental para controlar la intervención en la exposición (tipo de entrenador, cirujano versus estudiante de medicina), a pesar de no usarse un método de aleatorización ${ }^{16,17}$. La finalidad fue determinar, de manera fehaciente, el efecto del tipo de instructor en los resultados y las habilidades adquiridas al concluir el proceso. El modelo permite identificar diferencias entre las 
dos intervenciones, a pesar de no contar con un método de aleatorización para disminuir el sesgo de selección de los participantes.

Los hallazgos de este estudio podrían usarse en población lega. En un ensayo clínico controlado sobre la efectividad de diferentes intervenciones educativas para el control básico del sangrado, el $87,7 \%$ de los participantes pudo colocar adecuadamente un torniquete después del entrenamiento ${ }^{6}$. En otro estudio, se comparó el aprendizaje de técnicas para el control del sangrado, entre un público relacionado y otro no relacionado con cuidados de la salud, y se demostró que el aprendizaje podía ser homogéneo entre los dos grupos ${ }^{18}$. Sin embargo, existen problemas de validez externa de los resultados obtenidos, ya que los recursos a los que el público lego tiene acceso durante situaciones de emergencia, son diferentes a los que se ofrecen en el contexto controlado del curso. Por esto, centrar la atención solo en recursos como el torniquete o los vendajes hemostáticos para el control eficiente de la hemorragia, haría imposible usar las herramientas diseñadas dentro del curso para circunstancias reales.

Para que estas estrategias tengan impacto, es decir, contribuyan a disminuir la mortalidad secundaria a hemorragia por trauma, es imperativo empoderar al público en general para que pueda responder de manera efectiva y sepa cómo controlar la hemorragia, usando las herramientas estándar impartidas con el curso (torniquete y vendajes comerciales) o aquellas más asequibles, por ejemplo, las manos, trapos o prendas de vestir. Lo anterior, podría contraponerse con la experiencia de la maratón de Boston, en la cual se les aplicó un torniquete en la escena, a $83 \%$ de las víctimas con heridas en las extremidades inferiores y hemorragia. A pesar de que los datos fueron inadecuados para sacar conclusiones sobre su papel en el evento, es una práctica aceptable que podría salvar vidas en entornos urbanos donde, generalmente, el tiempo de transporte es corto ${ }^{19}$.

En países con recursos limitados es factible el entrenamiento para el control básico del sangra- do. Este puede ser eficaz impartido por instructores con un bagaje amplio en técnicas de control del sangrado, como también, por instructores con un menor nivel de formación, pero bien entrenados. En estudios futuros se debe ahondar en la viabilidad de implementar el entrenamiento de público lego con herramientas que sean más asequibles, pero igual de seguras, para obtener un control rápido de la hemorragia.

\section{Limitaciones}

Una de las limitaciones más notables en este estudio fue la pérdida del seguimiento para evaluar el aprendizaje en el periodo posterior al entrenamiento, en 2I participantes (7,92 \%); sin embargo, la ausencia de estos datos no afectó de manera significativa el análisis del efecto de la intervención en la muestra de población.

Otro factor que se debe considerar, es que las circunstancias en las que se llevan a cabo el entrenamiento y la evaluación, no logran reproducir completamente las situaciones reales, en las que factores como el caos y el estrés podrían tener impacto sobre la respuesta de los evaluados.

Además, podría haber limitaciones en cuanto a la validez externa, ya que la mayoría de los participantes estaban relacionados con el ámbito de la salud; por esto, sería importante evaluar el efecto de esta intervención en público lego, que son, generalmente, quienes se enfrentan con mayor frecuencia a estas casualidades.

\section{Conclusión}

La implementación de cursos formales para entrenar al público en el control básico del sangrado en casos de trauma, es factible en países de bajos a medianos ingresos. Esto se puede llevar a cabo de manera eficaz, tanto por instructores con un bagaje amplio en técnicas de control del sangrado como por instructores con menor nivel de formación, una vez hayan recibido el entrenamiento adecuado.

Conflicto de interés: ninguno 


\section{Referencias}

I. Teixeira PGR, Inaba K, Hadjizacharia P, Brown C, Sa$\lim \mathrm{A}$, Rhee $\mathrm{P}$, et al. Preventable or potentially preventable mortality at a mature trauma center. J Trauma. 2007;63:1338-47. doi: I0.I097/TA.obo13e3I8I5078ae

2. van Oostendorp SE, Tan EC, Geeraedts LM Jr. Prehospital control of life-threatening truncal and junctional haemorrhage is the ultimate challenge in optimizing trauma care; a review of treatment options and their applicability in the civilian trauma setting. Scand J Trauma Resusc Emerg Med. 2016;24:IIO. doi: IO.II86/ sI3049-0I6-030I-9

3. Sidwell RA, Spilman SK, Huntsman RS, Peláez CA. Efficient hemorrhage control skills training for healthcare employees. J Am Coll Surg. 20I8;226:160-4. doi: I0.IOI6/j.jamcollsurg.20I7.II.003

4. Warshaw AL. A systematic response to mass trauma: The public, organized first responders, and the American College of Surgeons. Bull Am Coll Surg. 2015;IOo(Suppl.):I4-5.

5. Bulger EM, Gestring ML, Jacobs LM. Optimizing bleeding control training for the public: A national imperative. JAMA Surg. 20I8;I53:799. doi: IO.IOoI/jamasurg.20I8.IIoo

6. Goralnick E, Chaudhary MA, Mccarty JC, Caterson EJ, Goldberg SA, Herrera-Escobar JP, et al. Effectiveness of instructional interventions for hemorrhage control readiness for laypersons in the Public Access and Tourniquet Training Study (PATTS): A randomized clinical trial. JAMA Surgery. 2018;02II5:I-9. doi: IO.IOOI/ jamasurg.2018.I099

7. Ordóñez CA. El cirujano de trauma y emergencias: pasado, presente y futuro. Rev Colomb Cir. 20I8;33:I726. doi: I0.30944/20II7582.43

8. Costa G. Citizen security in Latin America. InterAmerican Dialogue. 20I2;I-I4. Fecha de consulta: 24 de junio de 20I8. Disponible en: http://m.thedialogue. org/PublicationFiles/GinoCostaEnglishFINAL.PDF.

9. Pons PT, Jerome J, McMullen J, Manson J, Robinson J, Chapleau W. The Hartford consensus on active shooters: Implementing the continuum of prehospital trauma response. J Emerg Med. 2015;49:878-85. doi: I0.IoI6/j. jemermed.2015.09.013
Io. Rasmussen TE, Baer DG, Goolsby C. The giving back: Battlefield lesson to national preparedness. J Trauma Acute Care Surg. 20I6;80:I66-7. doi:Io.Io97/ TA.0000000000000899

II. Goolsby C, Jacobs L, Hunt RC, Goralnick E, Singletary EM, Levy MJ, et al. Stop the Bleed Education Consortium. J Trauma Acute Care Surg. 20I8;84:205-IO. doi: I0.I097/TA.ooooooooooooI732

I2. American College of Surgeons. Bleeding Control Basic (BCon) Course v. I.o.- BleedingControl.org. 2018. Fecha de consulta: I6 de enero de 20I7. Disponible en: https:// www.bleedingcontrol.org/private/materials.

13. A National Trauma Care System: Integrating military and civilian trauma systems to achieve zero preventable deaths after injury. Mil Med. 2017;182:1563-5. doi: I0.7205/MILMED-D-I7-00043

I4. Winkle RA. The effectiveness and cost effectiveness of public-access defibrillation. Clin Cardiol. 20I0;33:396-9. doi: wiley.com/I0.IO02/clc.20790

I5. Folke F, Lippert FK, Nielsen SL, Gislason GH, Hansen ML, Schramm TK, et al. Location of cardiac arrest in a city center: Strategic placement of automated external defibrillators in public locations. Circulation. 2009;I20:5IO-7. doi: /IO.II6I/CIRCULATIONAHA.IO8. 843755

I6. White H, Sabarwal S. Quasi-experimental design and methods. Florence: UNICEF Office of Research. Fecha de consulta: 27 de junio de 20I8. Disponible en: www. unicef-irc.org/KM/IE/img/downloads/Quasi-Experimental_Design_and_Methods_ENG.pdf.

I7. Harris AD, Mcgregor JC, Perencevich EN, Furuno JP, Zhu J, Peterson DE, et al. The use and interpretation of quasi-experimental studies in medical informatics. J Am Med Inform Assoc. 2006;13:I6-23. doi: I0.II97/jamia.Mr749

I8. Jacobs LM, Burns KJ. Tourniquet application training for individuals with and without a medical background in a hospital setting. J Trauma Acute Care Surg. 2015;78:442-5. doi: I0.I097/TA.0000000000000505

19. King DR, Larentzakis A, Ramly EP, Boston Trauma Collaborative. Tourniquet use at the Boston Marathon bombing: Lost in translation. J Trauma Acute Care Surg. 20I5;78:594-9. doi: I0.I097/TA.000ooooooooo056I 